\title{
Are There Some Premature Deaths We Should Not Prevent? Suicide Prevention when Assistance in Dying is Available
}

\author{
Brian L. Mishara
}

Brian Mishara is Professor of Psychology and Director of the Centre for Research and Intervention on Suicide and Euthanasia (CRISE) at the Université du Québec à Montréal, Canada. He is a founder of Suicide Action Montreal, the Montreal suicide prevention centre, a Vice-chairperson of the Trustees of Befrienders Worldwide, an international organization of helplines, a past president of the International Association for Suicide Prevention and Canadian Association for Suicide Prevention. He consults and conducts suicide prevention training internationally and has helped establish helplines using telephone, chat and text services. As he likes challenges, he is an active researcher in several fields, among which we should mention the effectiveness of suicide prevention programmes, understanding suicide in children, theories of suicidality, euthanasia etc. His recent research also focuses on best practices and ethical issues in using new technologies in suicide prevention.

Abstract. Suicide prevention services are generally supposed to do their utmost to prevent suicides with all persons, regardless of the suicidal individual's characteristics and reasons given for wanting to die. Their assumptions are that doing otherwise constitutes discrimination and that they would venture into an ethical morass if they attempt to determine whether some lives are more worthy of saving than others. However, where MAiD (assisted suicide and euthanasia) has been legalized, should we continue to strive to prevent all suicides? Or are there some circumstances where we should abstain from preventing a death by suicide or even encourage people to seek to end their lives by MAiD? This presentation explores if there are justified distinctions between how to respond to people requesting or considering requesting $\mathrm{MAiD}$, and how to respond to suicidal individuals. We examine whether suicide is sometimes rational and without ambivalence, as well as how respect for autonomy may be balanced against obligations to protect vulnerable populations. 